hep-ph/9709441

FTUV/97-44

IFIC/97-45

\title{
Weak Decays, Quark Mixing and CP Violation: Theory Overview*
}

\author{
A. Pich \\ Departament de Física Teòrica, IFIC, CSIC - Universitat de València, \\ Dr. Moliner 50, E-46100 Burjassot, València, Spain
}

\begin{abstract}
A brief overview of flavour-changing phenomena is presented. The main topics discussed are the universality and Lorentz structure of the leptonic charged-current couplings, our present knowledge of the quark-mixing matrix and the future prospects for $\mathrm{CP}$-violation studies.
\end{abstract}

\section{INTRODUCTION}

In spite of its enormous phenomenological success, the Standard Model (SM) leaves too many unanswered questions to be considered as a complete description of the fundamental forces. We do not understand yet why fermions are replicated in three (and only three) nearly identical copies? Why the pattern of masses and mixings is what it is? Are the masses the only difference among the three families? What is the origin of the SM flavour structure? Which dynamics is responsible for the observed CP violation?

The fermionic flavour is the main source of arbitrary free parameters in the SM: 9 fermion masses, 3 mixing angles and 1 complex phase (assuming the neutrinos to be massless). The problem of fermion-mass generation is deeply related with the mechanism responsible for the electroweak spontaneous symmetry breaking. Thus, the origin of these parameters lies in the most obscure part of the SM Lagrangian: the scalar sector. Clearly, the dynamics of flavour appears to be "terra incognita" which deserves a careful investigation.

The flavour structure looks richer in the quark sector, where mixing phenomena among the different families occurs (leptons would also mix if neutrino masses were non-vanishing). A precise measurement of the quark mixings would allow to test their predicted unitarity structure, and could give some hints about the unknown underlying dynamics. Since quarks are confined within hadrons, an accurate determination of their mix-

\footnotetext{
*Invited talk at the XVI International Workshop on Weak Interactions and Neutrinos (WIN'97), Capri, June 1997
}

ing parameters requires first a good understanding of hadronization effects in flavour-changing transitions. The interplay of strong interactions in weak decays plays a crucial role, which, unfortunately, is difficult to control due to the nonperturbative character of QCD at long distances.

In the SM flavour-changing transitions occur only in the charged-current sector:

$$
\begin{aligned}
& \mathcal{L}_{\mathrm{CC}}=\frac{g}{2 \sqrt{2}}\left\{W_{\mu}^{\dagger} J_{W}^{\mu}+\text { h.c. }\right\} \\
& J_{W}^{\mu}=\sum_{i j} \bar{u}_{i} \gamma^{\mu}\left(1-\gamma_{5}\right) \boldsymbol{V}_{i j} d_{j}+\sum_{l} \bar{\nu}_{l} \gamma^{\mu}\left(1-\gamma_{5}\right) l
\end{aligned}
$$

The so-called Cabibbo-Kobayashi-Maskawa [1,2 (CKM) matrix $\boldsymbol{V}$ couples any up-type quark with all down-type quarks. It originates from the same (unknown) Yukawa couplings giving rise to the quark masses.

For $N_{G}$ fermion generations, the quark-mixing matrix contains $\left(N_{G}-1\right)^{2}$ physical parameters: $N_{G}\left(N_{G}-1\right) / 2$ moduli and $\left(N_{G}-1\right)\left(N_{G}-2\right) / 2$ phases. In the simpler case of two generations, $\boldsymbol{V}$ is determined by a single parameter, the socalled Cabibbo angle [1]. With $N_{G}=3$, the CKM matrix is described by 3 angles and 1 phase [2]. This CKM phase is the only complex phase in the SM Lagrangian; thus, it is a unique source of $\mathrm{CP}$ violation. In fact, it was for this reason that the third generation was assumed to exist [2], before the discovery of the $\tau$ and the $b$. With two generations, the SM could not explain the observed CP violation in the $K$ system. 


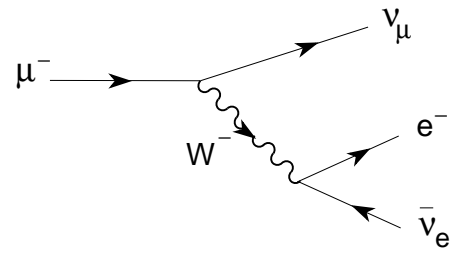

Figure 1. $\mu$-decay diagram.

\section{LEPTONIC DECAYS}

The simplest flavour-changing process is the leptonic decay of the muon, which proceeds through the $W$-exchange diagram shown in Fig. 1. The momentum transfer carried by the intermediate $W$ is very small compared to $M_{W}$. Therefore, the vector-boson propagator reduces to a contact interaction. The decay can then be described through an effective local 4-fermion Hamiltonian,

$\mathcal{H}_{\mathrm{eff}}=\frac{G_{F}}{\sqrt{2}}\left[\bar{e} \gamma^{\alpha}\left(1-\gamma_{5}\right) \nu_{e}\right]\left[\bar{\nu}_{\mu} \gamma_{\alpha}\left(1-\gamma_{5}\right) \mu\right]$

where $G_{F} / \sqrt{2}=g^{2} /\left(8 M_{W}^{2}\right)$ is called the Fermi coupling constant. $G_{F}$ is fixed by the total $\mu^{-}$ decay width,

$\Gamma\left(\mu^{-} \rightarrow e^{-} \bar{\nu}_{e} \nu_{\mu}\right)=\frac{G_{F}^{2} m_{\mu}^{5}}{192 \pi^{3}} f\left(m_{e}^{2} / m_{\mu}^{2}\right) r_{\mathrm{EW}}$,

where $f(x)=1-8 x+8 x^{3}-x^{4}-12 x^{2} \ln x$, and $r_{\mathrm{EW}}=0.9958$ takes into account the leading higher-order corrections [3]. The measured $\mu$ lifetime [4], implies the value $G_{F}=(1.16639 \pm$ $0.00002) \times 10^{-5} \mathrm{GeV}^{-2}$

\subsection{Lepton Universality}

The decays of the $\tau$ lepton proceed through the same $W$-exchange mechanism as the leptonic $\mu$ decay. The only difference is that several final states are kinematically allowed: $\tau^{-} \rightarrow \nu_{\tau} e^{-} \bar{\nu}_{e}$, $\tau^{-} \rightarrow \nu_{\tau} \mu^{-} \bar{\nu}_{\mu}, \tau^{-} \rightarrow \nu_{\tau} d \bar{u}$ and $\tau^{-} \rightarrow \nu_{\tau} s \bar{u}$. Owing to the universality of the $W$ couplings, all these decay modes have equal probabilities (if final fermion masses and QCD interactions are neglected), except for an additional $N_{C}\left|\boldsymbol{V}_{u i}\right|^{2}$ factor $(i=d, s)$ in the semileptonic channels, where $N_{C}=3$ is the number of quark colours.

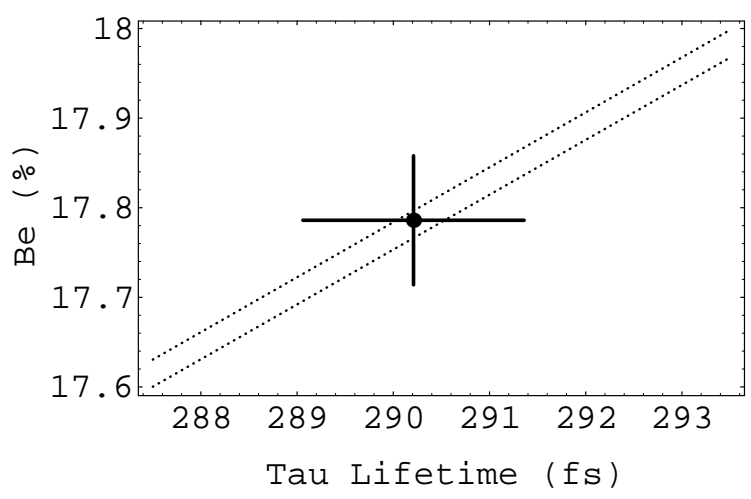

Figure 2. $B_{e} / \tau_{\tau}$ correlation [5]. The dotted band is the SM prediction in Eq. (A).

Using the value of $G_{F}$ measured in $\mu$ decay, Eq. (3) (with trivial kinematical changes) provides a relation [5] between the $\tau$ lifetime and the leptonic branching ratios $B_{l} \equiv B\left(\tau^{-} \rightarrow \nu_{\tau} l^{-} \bar{\nu}_{l}\right)$ :

$$
\begin{aligned}
B_{e} & =\frac{B_{\mu}}{0.972564 \pm 0.000010} \\
& =\frac{\tau_{\tau}}{(1.6321 \pm 0.0014) \times 10^{-12} \mathrm{~s}} .
\end{aligned}
$$

The predicted $B_{\mu} / B_{e}$ ratio is in perfect agreement with the measured value $B_{\mu} / B_{e}=0.974 \pm$ 0.006. As shown in Fig. 2, the relation between $B_{e}$ and $\tau_{\tau}$ is also well satisfied by the present data. These measurements test the universality of the $W$ couplings to the leptonic charged currents. The $B_{\mu} / B_{e}$ ratio constraints $\left|g_{\mu} / g_{e}\right|$, while $B_{e} / \tau_{\tau}$ provide information on $\left|g_{\tau} / g_{\mu}\right|$. The present results [5] are shown in Tables 1 and 2, together with the values obtained from the ra$\operatorname{tios} R_{\pi \rightarrow e / \mu} \equiv \Gamma\left(\pi^{-} \rightarrow e^{-} \bar{\nu}_{e}\right) / \Gamma\left(\pi^{-} \rightarrow \mu^{-} \bar{\nu}_{\mu}\right)$ and $R_{\tau / P} \equiv \Gamma\left(\tau^{-} \rightarrow \nu_{\tau} P^{-}\right) / \Gamma\left(P^{-} \rightarrow \mu^{-} \bar{\nu}_{\mu}\right)$ $[P=\pi, K]$, and from the comparison of the $\sigma \cdot B$ partial production cross-sections for the various $W^{-} \rightarrow l^{-} \bar{\nu}_{l}$ decay modes at the $p-\bar{p}$ colliders.

\subsection{Lorentz structure}

Let us consider the leptonic decay $l^{-} \rightarrow$ $\nu_{l} l^{\prime-} \bar{\nu}_{l^{\prime}}$. The most general, local, derivative-free, lepton-number conserving, four-lepton interaction Hamiltonian, consistent with locality and 
Table 1

Present constraints [5] on $\left|g_{\mu} / g_{e}\right|$.

\begin{tabular}{lc}
\hline & $\left|g_{\mu} / g_{e}\right|$ \\
\hline$B_{\mu} / B_{e}$ & $1.0005 \pm 0.0030$ \\
$R_{\pi \rightarrow e / \mu}$ & $1.0017 \pm 0.0015$ \\
$\sigma \cdot B_{W \rightarrow \mu / e}$ & $1.01 \pm 0.04$ \\
\hline
\end{tabular}

Table 2

Present constraints [5] on $\left|g_{\tau} / g_{\mu}\right|$.

\begin{tabular}{lc}
\hline & $\left|g_{\tau} / g_{\mu}\right|$ \\
\hline$B_{e} \tau_{\mu} / \tau_{\tau}$ & $1.0001 \pm 0.0029$ \\
$R_{\tau / \pi}$ & $1.005 \pm 0.005$ \\
$R_{\tau / K}$ & $0.984 \pm 0.020$ \\
$R_{\tau / h}$ & $1.004 \pm 0.005$ \\
$\sigma \cdot B_{W \rightarrow \tau / \mu}$ & $0.99 \pm 0.05$ \\
\hline
\end{tabular}

Lorentz invariance,

$\mathcal{H}=4 \frac{G_{l^{\prime} l}}{\sqrt{2}} \sum_{n, \epsilon, \omega} g_{\epsilon \omega}^{n}\left[\overline{l_{\epsilon}^{\prime}} \Gamma^{n}\left(\nu_{l^{\prime}}\right)_{\sigma}\right]\left[\overline{\left(\nu_{l}\right)_{\lambda}} \Gamma_{n} l_{\omega}\right]$,

contains ten complex coupling constants or, since a common phase is arbitrary, nineteen independent real parameters which could be different for each leptonic decay. The subindices $\epsilon, \omega, \sigma, \lambda$ label the chiralities (left-handed, right-handed) of the corresponding fermions, and $n$ the type of interaction: scalar $(I)$, vector $\left(\gamma^{\mu}\right)$, tensor $\left(\sigma^{\mu \nu} / \sqrt{2}\right)$. For given $n, \epsilon, \omega$, the neutrino chiralities $\sigma$ and $\lambda$ are uniquely determined.

Taking out a common factor $G_{l^{\prime} l}$, which is determined by the total decay rate, the coupling constants $g_{\epsilon \omega}^{n}$ are normalized to [6]

$1=\sum_{n, \epsilon, \omega}\left|g_{\epsilon \omega}^{n} / N^{n}\right|^{2}$,

where $N^{n}=2,1,1 / \sqrt{3}$ for $n=\mathrm{S}, \mathrm{V}, \mathrm{T}$. In the SM, $g_{L L}^{V}=1$ and all other $g_{\epsilon \omega}^{n}=0$.

The couplings $g_{\epsilon \omega}^{n}$ can be investigated through the measurement of the final charged-lepton distribution and with the inverse decay $\nu_{l^{\prime}} l \rightarrow l^{\prime} \nu_{l}$.
For $\mu$ decay, where precise measurements of the polarizations of both $\mu$ and $e$ have been performed, there exist [4 stringent upper bounds on the couplings involving right-handed helicities. These limits show nicely that the bulk of the $\mu$-decay transition amplitude is indeed of the predicted V-A type: $g_{L L}^{V}>0.96(90 \% \mathrm{CL})$. Improved measurements of the $\mu$-decay parameters will be performed at PSI and TRIUMPH [7].

The $\tau$-decay experiments are starting to provide useful information on the decay structure. Figure 3 shows the most recent limits obtained by CLEO [8]. The measurement of the $\tau$ polarization allows to bound those couplings involving an initial right-handed lepton; however, information on the final charged-lepton polarization is still lacking. Moreover, the measurement of the inverse decay $\nu_{\tau} l \rightarrow \tau \nu_{l}$, needed to separate the $g_{L L}^{S}$ and $g_{L L}^{V}$ couplings, looks far out of reach.

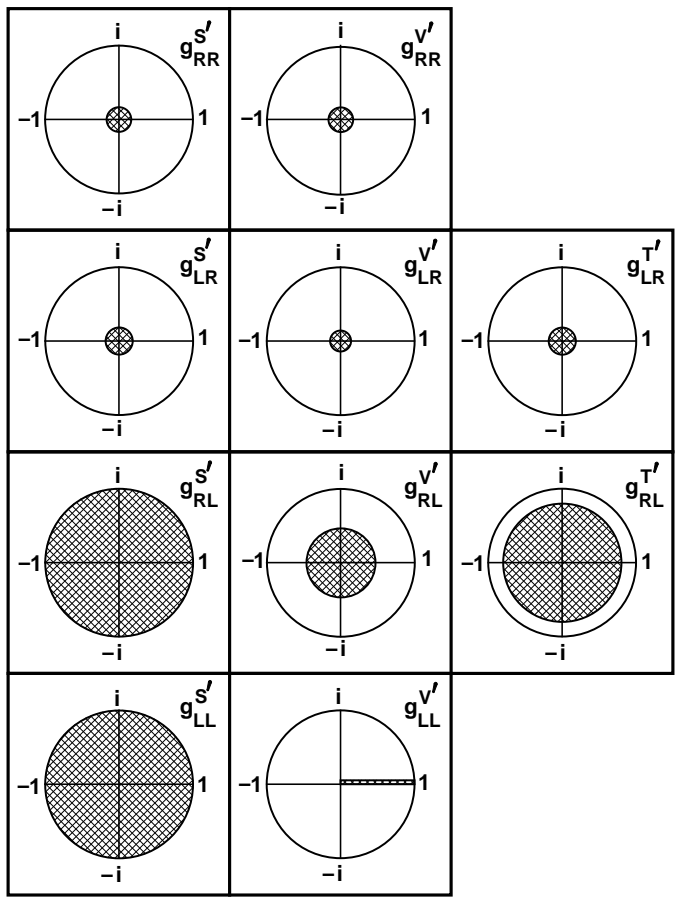

Figure 3. 90\% CL experimental limits [8] for the normalized $\tau$-decay couplings $g_{\epsilon \omega}^{\prime n} \equiv g_{\epsilon \omega}^{n} / N^{n}$, assuming $e / \mu$ universality. 


\section{SEMILEPTONIC DECAYS}

From now on we will assume that the chargedcurrent interaction is indeed universal and of the $\mathrm{V}-\mathrm{A}$ type, as predicted by the SM.

Let us consider the semileptonic weak decay $H \rightarrow H^{\prime} l^{-} \bar{\nu}_{l}$, associated with the corresponding quark transition $d_{j} \rightarrow u_{i} l^{-} \bar{\nu}_{l}$. The decay amplitude

$T \approx \frac{G_{F}}{\sqrt{2}} \boldsymbol{V}_{i j} \mathcal{M}_{H^{\prime} H}^{\mu} \bar{l} \gamma_{\mu}\left(1-\gamma_{5}\right) \nu_{l}$

involves an hadronic matrix element of the quark current: $\mathcal{M}_{H^{\prime} H}^{\mu} \equiv\left\langle H^{\prime}\left|\bar{u}_{i} \gamma^{\mu}\left(1-\gamma_{5}\right) d_{j}\right| H\right\rangle$. The evaluation of this matrix element is a nonperturbative QCD problem and, therefore, introduces unavoidable theoretical uncertainties.

Usually, one looks for a semileptonic transition where the matrix element can be fixed at some kinematical point, by a symmetry principle This has the virtue of reducing the theoretical uncertainties to the level of symmetry-breaking corrections and kinematical extrapolations. The standard example is a $0^{-} \rightarrow 0^{-}$decay such as $K \rightarrow \pi l \nu, D \rightarrow K l \nu$ or $B \rightarrow D l \nu$. Only the vector current can contribute in this case:

$\mathcal{M}_{P^{\prime} P}^{\mu} \sim\left(k+k^{\prime}\right)^{\mu} f_{+}\left(q^{2}\right)+\left(k-k^{\prime}\right)^{\mu} f_{-}\left(q^{2}\right)$,

where $q^{2}=\left(k-k^{\prime}\right)^{2}$ is the momentum transfer carried by the intermediate $W$. The unknown strong dynamics is fully contained in the two form factors $f_{ \pm}\left(q^{2}\right)$.

Since $\left(k-k^{\prime}\right)^{\mu} \bar{l} \gamma_{\mu}\left(1-\gamma_{5}\right) \nu_{l} \sim m_{l}$, the contribution of $f_{-}\left(q^{2}\right)$ is kinematically suppressed in the $e$ and $\mu$ modes. Moreover, there is an additional mass suppression of the $f_{-}\left(q^{2}\right)$ term for light quarks: $f_{-}\left(q^{2}\right) \approx\left(m_{u_{i}}-m_{d_{j}}\right)$. The decay width can then be written as

$\Gamma \approx \frac{G_{F}^{2} M_{P}^{5}}{192 \pi^{3}}\left|\boldsymbol{V}_{i j}\right|^{2}\left|f_{+}(0)\right|^{2} \mathcal{I}\left(1+\delta_{\mathrm{RC}}\right)$,

where $\delta_{\mathrm{RC}}$ is an electroweak radiative correction factor and $\mathcal{I}$ denotes a phase-space integral, which in the $m_{l}=0$ limit takes the form

$\mathcal{I} \approx \int \frac{d q^{2}}{M_{P}^{8}} \lambda^{3 / 2}\left(q^{2}, M_{P}^{2}, M_{P^{\prime}}^{2}\right)\left|\frac{f_{+}\left(q^{2}\right)}{f_{+}(0)}\right|^{2}$.

The usual procedure to determine $\left|\boldsymbol{V}_{i j}\right|$ involves three steps [9]:
1. Measure the shape of the $q^{2}$ distribution. This fixes the ratio $\left|f_{+}\left(q^{2}\right) / f_{+}(0)\right|$ and therefore determines $\mathcal{I}$.

2. Measure the total decay width $\Gamma$. Since $G_{F}$ is already known from $\mu$ decay, the product $\left|f_{+}(0)\right|\left|\boldsymbol{V}_{i j}\right|$ is determined.

3. Get a theoretical prediction for the normalization $f_{+}(0)$.

The important point to realize is that theoretical input is always needed. Thus, the accuracy of the $\left|\boldsymbol{V}_{i j}\right|$ determination is limited by our ability to calculate the relevant hadronic input.

The present (direct) determinations of the CKM matrix elements are summarized in Table 3. For light quarks (u, d, s), the chiral symmetry of massless QCD fixes the normalization of the relevant hadronic form factors at zero momentum transfer; moreover, symmetry-breaking corrections can be investigated rigorously with Chiral Perturbation Theory $(\chi \mathrm{PT})$ techniques [10]. Therefore, a rather good accuracy has been achieved. Note however, that there is a longstanding discrepancy $(\sim 2.5 \sigma)$ between the $\left|\boldsymbol{V}_{u d}\right|$ values obtained from nuclear $\beta$ decay and the neutron lifetime determination.

In the limit of infinite (c, b) quark masses the QCD Lagrangian has additional flavour and spin symmetries [11], which allow to fix the normalization of the $\mathcal{M}_{D B}^{\mu}$ and $\mathcal{M}_{D^{*} B}^{\mu}$ hadronic matrix elements at the point of zero recoil (maximum momentum transfer through the $W$ propagator). This point corresponds to the kinematical configuration where the initial and final mesons have identical velocities. Symmetry-breaking corrections can also be estimated with the methods of Heavy Quark Effective Theory (HQET) [12]. A reasonable determination of $\left|\boldsymbol{V}_{c b}\right|$ can then be obtained. Also shown in Table 3 is the determination of this CKM matrix element from the inclusive measurement of $\Gamma\left(b \rightarrow c l \bar{\nu}_{l}\right)$; although free from hadronic form factor uncertainties, this observable is very sensitive to the not so well-known values of the bottom and charm quark masses.

The remaining CKM determinations cannot make use of any useful symmetry to control the hadronization effects (the relevant quarks are too 
Table 3

Direct $\boldsymbol{V}_{i j}$ determinations.

\begin{tabular}{|c|c|c|c|}
\hline CKM entry & Value & Source & Symmetry \\
\hline \multirow[t]{2}{*}{$\left|\boldsymbol{V}_{u d}\right|$} & $0.9740 \pm 0.0005$ & Nuclear $\beta$ decay 13 & Isospin $(\chi \mathrm{PT})$ \\
\hline & $0.979 \pm 0.002$ & $n \rightarrow p e^{-} \bar{\nu}_{e}$, & Isospin $(\chi \mathrm{PT})$ \\
\hline \multirow[t]{2}{*}{$\left|\boldsymbol{V}_{u s}\right|$} & $0.2196 \pm 0.0023$ & $K_{e 3}$ & $\mathrm{SU}(3) \quad(\chi \mathrm{PT})$ \\
\hline & $0.222 \pm 0.003$ & Hyperon decays 幽 & $\mathrm{SU}(3) \quad(\chi \mathrm{PT})$ \\
\hline$\left|\boldsymbol{V}_{c d}\right|$ & $0.224 \pm 0.016$ & $\nu d \rightarrow c X$, 4] & - \\
\hline$\left|\boldsymbol{V}_{c s}\right|$ & $1.01 \pm 0.18$ & $D \rightarrow \bar{K} e^{+} \nu_{e}$ & - \\
\hline$\left|\boldsymbol{V}_{u b}\right|$ & $\left(3.3 \pm 0.2_{-0.4}^{+0.3} \pm 0.7\right) \times 10^{-3}$ & $B^{0} \rightarrow \pi^{-} l^{+} \nu_{l}, \rho^{-} l^{+} \nu_{l}$ 16] & - \\
\hline$\left|\boldsymbol{V}_{u b} / \boldsymbol{V}_{c b}\right|$ & $0.08 \pm 0.02$ & $b \rightarrow u l^{-} \bar{\nu}_{l}$ (end-point) 《] & - \\
\hline \multirow[t]{2}{*}{$\left|\boldsymbol{V}_{c b}\right|$} & $0.038 \pm 0.003$ & $B \rightarrow D^{*} l \bar{\nu}_{l}[17-19$ & $M_{b} \rightarrow \infty \quad(\mathrm{HQET})$ \\
\hline & $0.040 \pm 0.004$ & $b \rightarrow c l \bar{\nu}_{l} \quad$ (inclusive) [17, 18 & $M_{b} \rightarrow \infty \quad(\mathrm{HQET})$ \\
\hline$\frac{\left|\boldsymbol{V}_{t b}\right|}{\sqrt{\left|\boldsymbol{V}_{t d}\right|^{2}+\left|\boldsymbol{V}_{t s}\right|^{2}+\left|\boldsymbol{V}_{t b}\right|^{2}}}$ & $0.99 \pm 0.29$ & $t \rightarrow b W / q W$ 20] & - \\
\hline
\end{tabular}

heavy to consider the $m_{q} \rightarrow 0$ limit, and/or too light for the $m_{q} \rightarrow \infty$ approximation to make sense). Those determinations need to rely on explicit hadronic models; thus, the achievable precision is strongly limited by theoretical uncertainties. An obvious exception is the recent constraint on $\left|\boldsymbol{V}_{t b}\right|$, obtained at the Tevatron.

\subsection{CKM Unitarity}

The values of $\left|\boldsymbol{V}_{u i}\right|(i=d, s, b)$ provide a test of the unitarity of the CKM matrix:

$$
\left|\boldsymbol{V}_{u d}\right|^{2}+\left|\boldsymbol{V}_{u s}\right|^{2}+\left|\boldsymbol{V}_{u b}\right|^{2}=0.9973 \pm 0.0013 \text {. }
$$

To get this number, we have used the weighted average of the two $\left|\boldsymbol{V}_{u s}\right|$ determinations and the nuclear $\beta$ decay measurement of $\left|\boldsymbol{V}_{u d}\right|$. Given the disagreement with the neutron lifetime determination, it looks quite plausible that the small unitarity violation in Eq. (11) originates in the input $\left|\boldsymbol{V}_{u d}\right|$ value.

Assuming unitarity, a more precise picture of the mixings among the three quark generations is obtained [4]. The CKM matrix shows a hierarchical pattern, with the diagonal elements being very close to one, the ones connecting the two first generations having a size

$\lambda \equiv\left|\boldsymbol{V}_{u s}\right|=0.2205 \pm 0.0018$, the mixing between the second and third families being of order $\lambda^{2}$, and the mixing between the first and third quark flavours having a much smaller size of about $\lambda^{3}$. It is then quite practical to use the approximate parameterization [21]:

$\boldsymbol{V}=\left[\begin{array}{ccc}1-\frac{\lambda^{2}}{2} & \lambda & A \lambda^{3}(\rho-i \eta) \\ -\lambda & 1-\frac{\lambda^{2}}{2} & A \lambda^{2} \\ A \lambda^{3}(1-\rho-i \eta) & -A \lambda^{2} & 1\end{array}\right]$

valid up to $\mathcal{O}\left(\lambda^{4}\right)$ corrections. Here,

$A=\frac{\left|\boldsymbol{V}_{c b}\right|}{\lambda^{2}}=0.80 \pm 0.06$,

$\sqrt{\rho^{2}+\eta^{2}}=\left|\frac{\boldsymbol{V}_{u b}}{\lambda \boldsymbol{V}_{c b}}\right|=0.36 \pm 0.09$.

\section{NON-LEPTONIC TRANSITIONS}

The dynamical effect of the strong interaction is more important in non-leptonic transitions, where two different quark currents are involved and gluons can couple everywhere. Using the operator product expansion and renormalizationgroup techniques, these transitions can be described through effective Hamiltonians of the 
form

$\mathcal{H}_{\mathrm{eff}}=\sum_{i} C_{i}(\mu) Q_{i}$

with local four-fermion operators $Q_{i}$, modulated by Wilson coefficients $C_{i}(\mu)$. The arbitrary renormalization scale $\mu$ separates the short- $(M>\mu)$ and long- $(M<\mu)$ distance contributions, which are contained in $C_{i}(\mu)$ and $\left\langle Q_{i}\right\rangle(\mu)$ respectively. Thus, $C_{i}(\mu)$ contain all the information on CKM factors and heavy-mass scales. The physical amplitudes are of course independent of $\mu$.

A lot of effort has been invested recently in the calculation of Wilson coefficients at next-toleading order. For the most important processes, all contributions of $\mathcal{O}\left(\alpha_{s}^{n} L^{n}\right)$ and $\mathcal{O}\left(\alpha_{s}^{(n+1)} L^{n}\right)$ have been computed, where $L=\log (M / m)$ denotes the logarithm of any ratio of heavy-mass scales $(M, m \geq \mu)$. Moreover, the full $m_{t} / M_{W}$ dependence (at lowest order in $\alpha_{s}$ ) has been also included. A detailed summary of those calculations (with a complete list of references) can be found in Ref. 22].

Unfortunately, in order to predict the physical amplitudes one is still confronted with the calculation of the hadronic matrix elements $\left\langle Q_{i}\right\rangle(\mu)$ of the four-fermion operators $Q_{i}$. This is a very difficult non-perturbative problem which so far remains unsolved. We have only been able to obtain rough estimates using different approximations (vacuum saturation, $N_{C} \rightarrow \infty$ limit, QCD lowenergy effective action, ...) or applying QCD techniques (lattice, QCD sum rules) which suffer from their own technical limitations.

\section{5. $B^{0}-\bar{B}^{0}$ MIXING}

Additional information on the CKM parameters can be obtained from flavour-changing neutral-current transitions, occurring at the 1loop level. An important example is provided by the mixing between the $B^{0}$ meson and its antiparticle. This process occurs through the socalled box diagrams, where two $W$ bosons are exchanged between a pair of quark lines. The mixing amplitude is proportional to

$$
\left\langle\bar{B}_{d}^{0}\left|\mathcal{H}_{\Delta B=2}\right| B_{0}\right\rangle \sim \sum_{i j} \boldsymbol{V}_{i d} \boldsymbol{V}_{i b}^{*} \boldsymbol{V}_{j d}^{*} \boldsymbol{V}_{j b} S\left(r_{i}, r_{j}\right)
$$
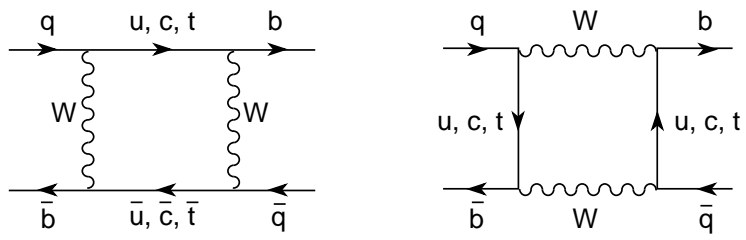

Figure 4. $B^{0}-\bar{B}^{0}$ mixing diagrams.

where $S\left(r_{i}, r_{j}\right)$ is a loop function which depends on the masses $\left[r_{i} \equiv m_{i}^{2} / M_{W}^{2}\right]$ of the up-type quarks running along the internal fermionic lines.

Owing to the unitarity of the CKM matrix, the mixing amplitude vanishes for equal (up-type) quark masses (GIM mechanism 23]); thus the effect is proportional to the mass splittings between the $u, c$ and $t$ quarks. Since the different CKM factors have all a similar size, $\boldsymbol{V}_{u d} \boldsymbol{V}_{u b}^{*} \sim$ $\boldsymbol{V}_{c d} \boldsymbol{V}_{c b}^{*} \sim \boldsymbol{V}_{t d} \boldsymbol{V}_{t b}^{*} \sim A \lambda^{3}$, the final amplitude is completely dominated by the top contribution; i.e. $\left\langle\bar{B}_{d}^{0}\left|\mathcal{H}_{\Delta B=2}\right| B_{0}\right\rangle \sim\left|\boldsymbol{V}_{t d}\right|^{2} S\left(r_{t}, r_{t}\right)$. This transition can then be used to perform an indirect determination of $\left|\boldsymbol{V}_{t d}\right|$.

The main theoretical uncertainty stems from the hadronic matrix element of the $\Delta B=2$ fourquark operator generated by the box diagrams:

$\left\langle\bar{B}^{0}\left|\left(\bar{b}_{L} \gamma^{\mu} d_{L}\right)\left(\bar{b}_{L} \gamma_{\mu} d_{L}\right)\right| B^{0}\right\rangle \equiv \frac{2}{3} M_{B}^{2}\left(\sqrt{2} \xi_{B}\right)^{2}$.

The size of this matrix element is characterized through the non-perturbative parameter $\xi_{B} \equiv$ $f_{B} \sqrt{\hat{B}_{B}}$, which is rather badly known. Lattice calculations give 24 $\sqrt{2} \xi_{B}=207 \pm 30 \mathrm{MeV}$, while QCD sum rules provide a slightly larger (but consistent) value, $\sqrt{2} \xi_{B}=260 \pm 70 \mathrm{MeV}$ 25. Taking the range $\sqrt{2} \xi_{B}=215 \pm 40 \mathrm{MeV}$, the measured mixing between the $B_{d}^{0}-\bar{B}_{d}^{0}$ mesons, $\Delta M_{B_{d}^{0}}=0.463 \pm 0.018 \mathrm{ps}^{-1}$ 18, 26, implies:

$\left|\boldsymbol{V}_{t d}\right|=0.0080 \pm 0.0003_{-0.0013}^{+0.0018}$,

where the first error is the experimental one and the second reflects the theoretical uncertainties.

In terms of the $(\rho, \eta)$ parameterization, this gives

$\sqrt{(1-\rho)^{2}+\eta^{2}}=\left|\frac{\boldsymbol{V}_{t d}}{\lambda \boldsymbol{V}_{c b}}\right|=0.93_{-0.17}^{+0.22}$. 
A similar analysis can be applied to the $B_{s}^{0-}$ $\bar{B}_{s}^{0}$ mixing probability. The non-perturbative uncertainties can be reduced to the level of $S U(3)$ breaking corrections through the ratio

$$
\frac{\Delta M_{B_{s}^{0}}}{\Delta M_{B_{d}^{0}}} \approx \frac{M_{B_{s}^{0}} \xi_{B_{s}^{0}}^{2}}{M_{B_{d}^{0}} \xi_{B_{d}^{0}}^{2}}\left|\frac{\boldsymbol{V}_{t s}}{\boldsymbol{V}_{t d}}\right|^{2} \equiv \Omega \times\left|\frac{\boldsymbol{V}_{t s}}{\boldsymbol{V}_{t d}}\right|^{2} .
$$

Taking $\Omega \approx 1.15 \pm 0.15$, the present bound $\Delta M_{B_{s}^{0}}>10.2 \mathrm{ps}^{-1}(95 \% \mathrm{CL})$ 18.27 implies

$$
\left|\frac{\boldsymbol{V}_{t s}}{\boldsymbol{V}_{t d}}\right| \approx \frac{1}{\lambda \sqrt{(1-\rho)^{2}+\eta^{2}}}>3.8 \text {. }
$$

\section{CP VIOLATION}

With only two fermion generations the quarkmixing mechanism cannot give rise to $\mathrm{CP}$ violation; therefore, for $\mathrm{CP}$ violation to occur in a particular process, all 3 generations are required to play an active role. In the kaon system, for instance, $\mathrm{CP}$-violation effects can only appear at the one-loop level, where the top quark is present. In addition, all CKM matrix elements must be non-zero and the quarks of a given charge must be non-degenerate in mass. If any of these conditions were not satisfied, the CKM phase could be rotated away by a redefinition of the quark fields. $\mathrm{CP}$-violation effects are then necessarily proportional to the product of all CKM angles, and should vanish in the limit where any two (equal-charge) quark masses are taken to be equal. Thus, violations of the CP symmetry are necessarily small.

Up to know, the only experimental evidence of $\mathrm{CP}$-violation phenomena comes from the kaon system. The ratios,

$$
\begin{gathered}
\eta_{+-} \equiv \frac{A\left(K_{L} \rightarrow \pi^{+} \pi^{-}\right)}{A\left(K_{S} \rightarrow \pi^{+} \pi^{-}\right)} \approx \varepsilon_{K}+\varepsilon_{K}^{\prime}, \\
\eta_{00} \equiv \frac{A\left(K_{L} \rightarrow \pi^{0} \pi^{0}\right)}{A\left(K_{S} \rightarrow \pi^{0} \pi^{0}\right)} \approx \varepsilon_{K}-2 \varepsilon_{K}^{\prime},
\end{gathered}
$$

involve final $2 \pi$ states which are even under CP. Therefore, they measure a $\mathrm{CP}$-violating amplitude which can originate either from a small CPeven admixture in the initial $K_{L}$ state (indirect $\mathrm{CP}$ violation), parameterized by $\varepsilon_{K}$, or from direct $\mathrm{CP}$ violation in the decay amplitude. This latter effect, parameterized by $\varepsilon_{K}^{\prime}$, requires the interference between the two $K \rightarrow 2 \pi$ isospin $(I=0,2)$ amplitudes, with different weak and strong phases.

The parameter $\varepsilon_{K}$ is well determined [4]:

$\varepsilon_{K}=(2.280 \pm 0.013) \times 10^{-3} \mathrm{e}^{i \phi\left(\varepsilon_{K}\right)}$,

$\phi\left(\varepsilon_{K}\right) \approx \arctan \left(\frac{-2 \Delta M_{K^{0}}}{\Delta \Gamma_{K^{0}}}\right)=43.49^{\circ} \pm 0.08^{\circ}$.

$\varepsilon_{K}$ has been also measured [4] through the $\mathrm{CP}$ asymmetry between the two $K_{L} \rightarrow$ $\pi^{\mp} l^{ \pm} \stackrel{(-)}{\nu}$ semileptonic decay widths, which implies $\operatorname{Re}\left(\varepsilon_{K}\right)=(1.63 \pm 0.06) \times 10^{-3}$, in good agreement with (22).

The value of $\varepsilon_{K}^{\prime}$ is not so-well established. Two different experiments have recently reported a measurement of this quantity:

$\operatorname{Re}\left(\frac{\varepsilon_{K}^{\prime}}{\varepsilon_{K}}\right)=\left\{\begin{array}{ll}(23.0 \pm 6.5) \times 10^{-4} & {[\mathrm{NA} 31]} \\ (7.4 \pm 5.9) \times 10^{-4} & {[\mathrm{E} 731]}\end{array}\right.$.

The NA31 measurement 28] provides evidence for a non-zero value of $\varepsilon_{K}^{\prime} / \varepsilon_{K}$ (i.e., direct $\mathrm{CP}$ violation), with a statistical significance of more than three standard deviations. However, this is not supported by the E731 result 29], which is compatible with $\varepsilon_{K}^{\prime} / \varepsilon_{K}=0$, thus with no direct $\mathrm{CP}$ violation. The probability for the two results being statistically compatible is only $7.6 \%$.

The next generation of $\varepsilon_{K}^{\prime} / \varepsilon_{K}$ experiments is already ready at CERN (NA48 [30) and Fermilab (KTEV [31]). Moreover, a dedicated $\phi$ factory (DA $\Phi \mathrm{NE}$ ), providing large amounts of tagged $K_{S}, K_{L}$ and $K^{ \pm}(\phi \rightarrow K \bar{K})$, will start running soon at Frascati 32]. The goal of all these experiments is to reach sensitivities better than $10^{-4}$.

The CKM mechanism generates $\mathrm{CP}$-violation effects both in the $\Delta S=2 K^{0}-\bar{K}^{0}$ transition (box diagrams) and in the $\Delta S=1$ decay amplitudes (penguin diagrams). The theoretical analysis of $K^{0}-\bar{K}^{0}$ mixing is quite similar to the one applied to the $B$ system. This time, however, the charm loop contributions are non-negligible. The main uncertainty stems from the calculation of the hadronic matrix element of the four-quark $\Delta S=2$ operator, which is usually parameter- 


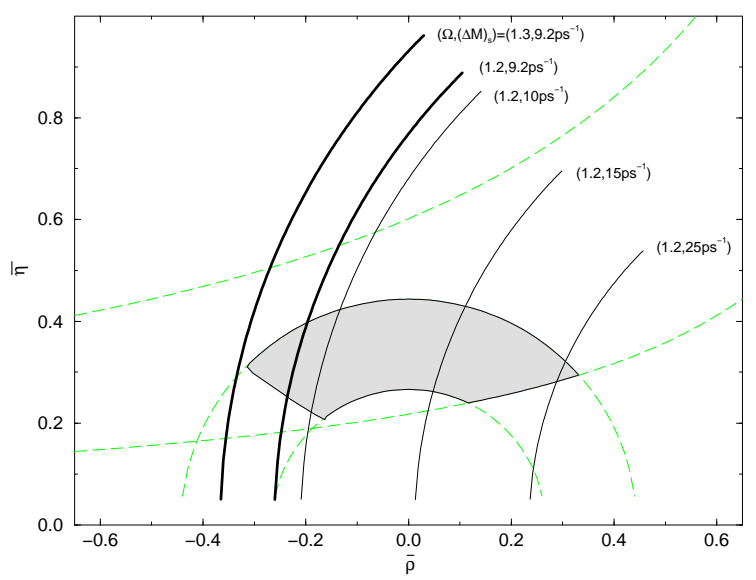

Figure 5. Unitarity Triangle constraints 22].

ized through the non-perturbative parameter 25] $\hat{B}_{K} \approx 0.4-0.8$.

The experimental value of $\varepsilon_{K}$ specifies a hyperbola in the $(\rho, \eta)$ plane. This is shown in Fig. 5, together with the constraints obtained from $\left|\boldsymbol{V}_{u b} / \boldsymbol{V}_{c b}\right|$ and $B_{d}^{0}-\bar{B}_{d}^{0}$ mixing. This figure, taken from Ref. [22], assumes $\hat{B}_{K}=0.75 \pm 0.15$ and $\sqrt{2} \xi_{B}=200 \pm 40 \mathrm{MeV}$. Also shown in the figure is the impact of the experimental bound on $\Delta M_{B_{s}^{0}}$.

The theoretical estimate of $\varepsilon_{K}^{\prime} / \varepsilon_{K}$ is much more involved, because ten four-quark operators need to be considered in the analysis and the presence of cancellations between different contributions tends to amplify the sensitivity to the not very well controlled long-distance effects. For large values of the top mass, the $Z^{0}$-penguin contributions strongly suppress the expected value of $\varepsilon_{K}^{\prime} / \varepsilon_{K}$, making the final result very sensitive to $m_{t}$. The present theoretical estimates [22] range from $-1.2 \times 10^{-4}$ to $1.6 \times 10^{-3}$. More theoretical work is needed in order to get firm predictions.

\subsection{The Unitarity Triangle}

The SM mechanism of $\mathrm{CP}$ violation is based in the unitarity of the CKM matrix. Up to now, the only unitarity relation which has been precisely tested is the one associated with the first row; however, only the moduli of the CKM parameters appear in Eq. (11), while $\mathrm{CP}$ violation has to do with their phases. The most interesting off-diagonal unitarity condition is

$\boldsymbol{V}_{u b}^{*} \boldsymbol{V}_{u d}+\boldsymbol{V}_{c b}^{*} \boldsymbol{V}_{c d}+\boldsymbol{V}_{t b}^{*} \boldsymbol{V}_{t d}=0$,

which involves three terms of similar size. This relation can be visualized by a triangle in the complex plane, which is usually scaled by dividing its sides by $\boldsymbol{V}_{c b}^{*} \boldsymbol{V}_{c d}$. This aligns one side of the triangle along the real axis and makes its length equal to 1 ; the coordinates of the 3 vertices are then $(0,0),(1,0)$ and $(\rho, \eta)$. In the absence of $\mathrm{CP}$ violation, this unitarity triangle would degenerate into a segment along the real axis.

Although the orientation of the triangle in the complex plane is phase-convention dependent, the triangle itself is a physical object: the length of the sides and the angles $(\alpha, \beta, \gamma)$ can be directly measured. In fact, we have already determined its sides from $\Gamma(b \rightarrow u) / \Gamma(b \rightarrow c)$ and $B_{d}^{0-}$ $\bar{B}_{d}^{0}$ mixing, and the position of the $(\rho, \eta)$ vertex has been further pinned down with the additional information provided by $\varepsilon_{K}$.

\section{FUTURE PROSPECTS}

\subsection{Bottom Decays}

The flavour-specific decays $B^{0} \rightarrow X l^{+} \nu_{l}$ and $\bar{B}^{0} \rightarrow X l^{-} \bar{\nu}_{l}$ provide the most direct way to measure the amount of $\mathrm{CP}$ violation in the $B^{0}-\bar{B}^{0}$ mixing matrix, through the asymmetry between the number of $l^{+} l^{+}$and $l^{-} l^{-}$pairs produced in the processes $e^{+} e^{-} \rightarrow B^{0} \bar{B}^{0} \rightarrow l^{ \pm} l^{ \pm} X$. This $\Delta B=2$ asymmetry is expected to be quite tiny in the SM, because $\left|\Delta \Gamma_{B^{0}} / \Delta M_{B^{0}}\right| \sim m_{b}^{2} / m_{t}^{2}<<$ 1 ; moreover, there is an additional GIM suppression $\sim\left(m_{c}^{2}-m_{u}^{2}\right) / m_{b}^{2}$. The observation of an asymmetry at the percent level, would be a clear indication of new physics beyond the SM.

Direct CP violation could be established by measuring a non-zero rate asymmetry in $B^{ \pm}$decays. Unfortunately, the necessary presence of a strong-phase difference makes difficult to obtain clean information on the CKM matrix from this kind of observables.

The large $B^{0}-\bar{B}^{0}$ mixing provides a different way to generate the required $\mathrm{CP}$-violating interference. There are quite a few non-leptonic final states which are reachable both from a $B^{0}$ and a 
$\bar{B}^{0}$. For these flavour non-specific decays the $B^{0}$ (or $\bar{B}^{0}$ ) can decay directly to the given final state $f$, or do it after the meson has been changed to its antiparticle via the mixing process; i.e., there are two different amplitudes, $A\left(B^{0} \rightarrow f\right)$ and $A\left(B^{0} \rightarrow \bar{B}^{0} \rightarrow f\right)$, corresponding to two possible decay paths. $\mathrm{CP}$-violating effects can then result from the interference of these two contributions.

$B^{0}$ decays into $\mathrm{CP}$ self-conjugate final states are particularly promising. In that case, assuming that only one weak amplitude contributes to the $B^{0} \rightarrow f$ transition, all dependence on strong interaction effects disappears from the $\mathrm{CP}$-violating rate asymmetries. Therefore, they could provide a direct and clean measurement of the CKM parameters. The angles of the unitarity triangle could be directly determined through the decay modes $B_{d}^{0} \rightarrow J / \psi K_{S}(\beta), B_{d}^{0} \rightarrow \pi^{+} \pi^{-}$ $(\beta+\gamma=\pi-\alpha)$ and $B_{s}^{0} \rightarrow \rho^{0} K_{S}(\gamma)$.

The crucial assumption is that only one weak amplitude contributes to a given decay, which obviously is not the case; the (usually) dominant $\mathrm{W}$-exchange decay amplitude gets corrected by diagrams with different CKM structure, such as the so-called penguins. The gold-plated exception is $B_{d}^{0} \rightarrow J / \psi K_{S}$, since all decay amplitudes share the same dependence on CKM factors to an excellent approximation; thus, it will provide a very clean measurement of $\beta \equiv$ $-\arg \left(\boldsymbol{V}_{c d} \boldsymbol{V}_{c b}^{*} / \boldsymbol{V}_{t d} \boldsymbol{V}_{t b}^{*}\right)$.

In the case of $B_{d}^{0} \rightarrow \pi^{+} \pi^{-}$, penguin diagrams generate indeed a different CKM dependence, but they are numerically suppressed allowing for an approximate determination of $\alpha \equiv$ $-\arg \left(\boldsymbol{V}_{t d} \boldsymbol{V}_{t b}^{*} / \boldsymbol{V}_{u d} \boldsymbol{V}_{u b}^{*}\right)$. The measurement of $\gamma$ with $B_{s}^{0} \rightarrow \rho^{0} K_{S}$ is however not feasible; the $d i$ rect decay amplitude is colour suppressed, leading presumably to a large (maybe dominant) penguin contamination.

Many additional tests of the CKM matrix with $B$ decays have been proposed. The rich variety of available decay modes provides ways to circumvent the strong interaction complications through relations (isospin, $\mathrm{SU}(3), D^{0}-\bar{D}^{0}$ mixing) among different processes or measuring the time evolution. A detailed summary of recent work can be found in Ref. 22].

\subsection{Rare K Decays}

The decay $K^{+} \rightarrow \pi^{+} \nu \bar{\nu}$ is a well-known example of an allowed process where long-distance effects play a negligible role. Thus, this mode provides a good test of the CKM structure. The decay process is dominated by short-distance loops ( $Z$ penguin, $W$ box) involving the heavy top quark, but receives also sizeable contributions from internal charm-quark exchanges. The resulting decay amplitude is proportional to the hadronic matrix element of the $\Delta S=1$ vector current, which (assuming isospin symmetry) can be obtained from $K_{l 3}$ decays.

The branching ratio is predicted to be in the range [22] $\mathrm{Br} \sim(9.1 \pm 3.2) \times 10^{-11}$, to be compared with the recently reported signal (1 event) [33]:

$\operatorname{Br}\left(K^{+} \rightarrow \pi^{+} \nu \bar{\nu}\right)=\left(4.2_{-3.5}^{+9.7}\right) \times 10^{-10}$.

The $\mathrm{CP}$-violating decay $K_{L} \rightarrow \pi^{0} \nu \bar{\nu}$ has been suggested [34] as a good candidate to look for pure direct $\mathrm{CP}$-violating transitions. The contribution coming from indirect $\mathrm{CP}$ violation via $\mathrm{K}^{0}$ $\bar{K}^{0}$ mixing is very small $\left[34\right.$ : $\left.\operatorname{Br}\right|_{\varepsilon} \sim 5 \times 10^{-15}$. The decay proceeds almost entirely through direct $\mathrm{CP}$ violation (via interference with mixing), and is completely dominated by short-distance loop diagrams with top-quark exchanges [22]:

$\operatorname{Br}\left(K_{L} \rightarrow \pi^{0} \nu \bar{\nu}\right) \approx 8.07 \times 10^{-11} A^{4} \eta^{2} r_{t}^{1.15}$.

The present experimental upper bound [35], $\operatorname{Br}\left(K_{L} \rightarrow \pi^{0} \nu \bar{\nu}\right)<5.8 \times 10^{-5}(90 \% \mathrm{CL})$, is still far away from the expected range [22]

$\operatorname{Br}\left(K_{L} \rightarrow \pi^{0} \nu \bar{\nu}\right)=(2.8 \pm 1.7) \times 10^{-11}$.

Nevertheless, the experimental prospects to reach the required sensitivity in the near future look rather promising [36]. The clean observation of just a single unambiguous event would indicate the existence of $\mathrm{CP}$-violating $\Delta S=1$ transitions.

Another promising mode is $K_{L} \rightarrow \pi^{0} e^{+} e^{-}$. Owing to the electromagnetic suppression of the $2 \gamma \mathrm{CP}$-conserving contribution, this decay seems to be dominated by the $\mathrm{CP}$-violating one-photon emission amplitude. Moreover, the direct $\mathrm{CP}-$ violating contribution is expected to be larger than the indirect one [37].

A recent overview of many other interesting rare $\mathrm{K}$ decays can be found in Ref. [37]. 


\section{SUMMARY}

The flavour structure of the SM is one of the main pending questions in our understanding of weak interactions. Although we do not know the reason of the observed family replication, we have learnt experimentally that the number of SM generations is just three (and no more). Therefore, we must study as precisely as possible the few existing flavours, to get some hints on the dynamics responsible for their observed structure.

The SM incorporates a mechanism to generate $\mathrm{CP}$ violation, through the single phase naturally occurring in the CKM matrix. This mechanism, deeply rooted into the unitarity structure of $\boldsymbol{V}$, implies very specific requirements for $\mathrm{CP}$ violation to show up, which should be tested in appropriate experiments. The tiny $\mathrm{CP}$ asymmetry observed in the $\mathrm{K}$ system, can be parameterized through the CKM phase; however, we do not have yet an experimental verification of the CKM mechanism. A fundamental explanation of the origin of this phenomena is also lacking.

New and powerful flavour factories will become operational very soon. Many interesting $\mathrm{CP}-$ violation signals are expected to be seen in the near future. Large surprises may well be discovered, probably giving the first hints of new physics and offering clues to the problems of fermionmass generation, quark mixing and family replication.

\section{REFERENCES}

1. N. Cabibbo, Phys. Rev. Lett. 10 (1963) 531.

2. M. Kobayashi and T. Maskawa, Progr. Theor. Phys. 42 (1973) 652.

3. T. Kinoshita and A. Sirlin, Phys. Rev. 113 (1959) 1652.

4. Particle Data Group, Review of Particle Physics, Phys. Rev. D54 (1996) 1.

5. A. Pich, Nucl. Phys. B (Proc. Suppl.) 55C (1997) 3.

6. W. Fetscher, H.-J. Gerber and K.F. Johnson, Phys. Lett. B173 (1986) 102.

7. D. Guill, these proceedings.

8. J.P. Alexander et al, hep-ex/9705009.

9. A. Pich, hep-ph/9601202.
10. A. Pich, Rep. Prog. Phys. 58 (1995) 563.

11. N. Isgur and M. Wise, Phys. Lett. B232 (1989) 113; B237 (1990) 527.

12. M. Neubert, Phys. Rep. 245 (1994) 259.

13. E. Hagberg et al, nucl-ex/9609002.

14. W.J. Marciano, Ann. Rev. Nucl. Part. Sci. 41 (1991) 469.

15. H. Leutwyler and M. Roos, Z. Phys. C25 (1984) 91.

16. J.P. Alexander et al, Phys. Rev. Lett. 77 (1996) 5000.

17. M. Neubert, hep-ph/9702375.

18. K. Berkelman, these proceedings.

19. N. Marinelli, these proceedings.

20. D.W. Gerdes, hep-ex/9706001;

H.B. Greenlee, these proceedings.

21. L. Wolfenstein, Phys. Rev. Lett. 51 (1983) 1945.

22. A.J. Buras and R. Fleischer, hep-ph/9704376.

23. S.L. Glashow, J. Iliopoulos and L. Maiani, Phys. Rev. D2 (1970) 1285.

24. J. Flynn, Proc. 28th Int. Conf. on High Energy Physics (Warsaw, 1996), eds. Z. Ajduk and A.K. Wroblewski (World Scientific, Singapore, 1997) p. 335.

25. A. Pich and J. Prades, Phys. Lett. B346 (1995) 342.

26. E. Barberio, these proceedings.

27. G. Crawford, these proceedings.

28. G.D. Barr et al, Phys. Lett. B317 (1993) 233; H. Burkhardt et al, Phys. Lett. B206 (1988) 169.

29. L.K. Gibbons et al, Phys. Rev. Lett. 70 (1993) 1203.

30. P. Debu, these proceedings.

31. R. B. David, these proceedings.

32. G. De Zorzi, these proceedings.

33. S. Adler et al, hep-ex/9708031.

34. L.S. Littenberg, Phys. Rev. D39 (1989) 3322.

35. M. Weaver et al, Phys. Rev. Lett. 72 (1994) 3758 .

36. S. Adler, these proceedings.

37. A. Pich, Proc. of the Workshop on K Physics (Orsay, 1996), ed. L. Iconomidou-Fayard (Ed. Frontières, Gif-sur-Yvette, 1997) p. 353. 